\title{
Epigenetic Therapies for Heart Failure: Current Insights and Future Potential
}

\author{
Claudio Napoli ${ }^{1}$ \\ Paola Bontempo ${ }^{2}$ \\ Vittorio Palmieri ${ }^{3}$ \\ Enrico Coscioni $\mathbb{D D}^{4}$ \\ Ciro Maiello ${ }^{5}$ \\ Francesco Donatelli $\mathbb{D D}^{6}$ \\ Giuditta Benincasa' \\ 'Department of Advanced Medical and \\ Surgical Sciences (DAMSS), University of \\ Campania "Luigi Vanvitelli", Naples, \\ 80138, Italy; ${ }^{2}$ Department of Precision \\ Medicine, University of Campania "Luigi \\ Vanvitelli”, Naples, 80I38, Italy; \\ ${ }^{3}$ Department of Cardiac Surgery and \\ Transplantation, Heart Transplantation \\ Unit in Adults of the 'Ospedali dei Colli \\ Monaldi-Cotugno-CTO', Naples, Italy; \\ ${ }^{4}$ Department of Cardiac Surgery, Azienda \\ Ospedaliera Universitaria San Giovanni di \\ Dio e Ruggi d'Aragona, Salerno, Italy; \\ ${ }^{5}$ Department of Cardiovascular Surgery \\ and Transplants, Monaldi Hospital, \\ Azienda dei Colli, Naples, Italy; ${ }^{6}$ Chair of \\ Cardiac Surgery, Department of \\ Cardiothoracic Center, Istituto Clinico \\ Sant'Ambrogio, University of Milan, \\ Milan, Italy
}

\begin{abstract}
Despite the current reductionist approach providing an optimal indication for diagnosis and treatment of patients with heart failure with reduced ejection fraction (HFrEF), there are no standard pharmacological therapies for heart failure with preserved ejection fraction (HFpEF). Although in its infancy in cardiovascular diseases, the epigenetic-based therapy ("epidrugs") is capturing the interest of physician community. In fact, an increasing number of controlled clinical trials is evaluating the putative beneficial effects of: 1) direct epigenetic-oriented drugs, eg, apabetalone, and 2) repurposed drugs with a possible indirect epigenetic interference, eg, metformin, statins, sodium glucose transporter inhibitors 2 (SGLT2i), and omega 3 polyunsaturated fatty acids (PUFAs) in both HFrEF and HFpEF, separately. Apabetalone is the first and unique direct epidrug tested in cardiovascular patients to date, and the BETonMACE trial has reported a reduction in first HF hospitalization (any EF value) and cardiovascular death in patients with type 2 diabetes and recent acute coronary syndrome, suggesting a possible role in secondary prevention. Patients with HFpEF seem to benefit from supplementation to the standard therapy with statins, metformin, and SGLT2i owing to their ability in reducing mortality. In contrast, the vasodilator hydralazine, with or without isosorbide dinitrate, did not provide beneficial effects. In HFrEF, metformin and SGLT2 $\mathrm{i}$ could reduce the risk of incident HF and mortality in affected patients whereas clinical trials based on statins provided mixed results. Furthermore, PUFAs diet supplementation was significantly associated with reduced cardiovascular risk in both HFpEF and HFrEF. Future large trials will reveal whether direct and indirect epitherapy will remain a work in progress or become a useful way to customize the therapy in the real-world management of HFpEF and HFrEF. Our goal is to discuss the recent advancement in the epitherapy as a possible way to improve personalized therapy of HF.
\end{abstract}

Keywords: heart failure, personalized therapy, epidrugs

\section{Introduction}

Although novel drugs have successfully entered the clinical arena of heart failure with reduced ejection fraction (HFrEF), such as the PARADIGM-HF-derived angiotensin receptor neprilysin inhibitor (ARNI), disease-modifying therapies with a prognostic impact for patients affected by heart failure with preserved ejection fraction (HFpEF) are still lacking. ${ }^{1-5} \mathrm{HF}$ is a complex and highly prevalent syndrome for which the heart undergoes a substantial structural remodeling in patients at risk for major cardiovascular diseases (CVDs) (Figure 1). ${ }^{1-6}$ Gene-environment interactions can be mediated by specific patterns of epigenetic-sensitive changes (mainly DNA methylation and histone modifications) which may modulate the individual responsiveness to $\mathrm{HF}$ development. $^{6-14}$ This complex molecular circuit seems to trigger early cardiomyocyte loss, cardiac-remodeling, and micro- and macrovascular damage contributing to the
Correspondence: Giuditta Benincasa Department of Advanced Medical and Surgical Sciences (DAMSS), University of Campania "Luigi Vanvitelli", Naples, Italy Tel +390815667916

Email giuditta.benincasa@unicampania.it 


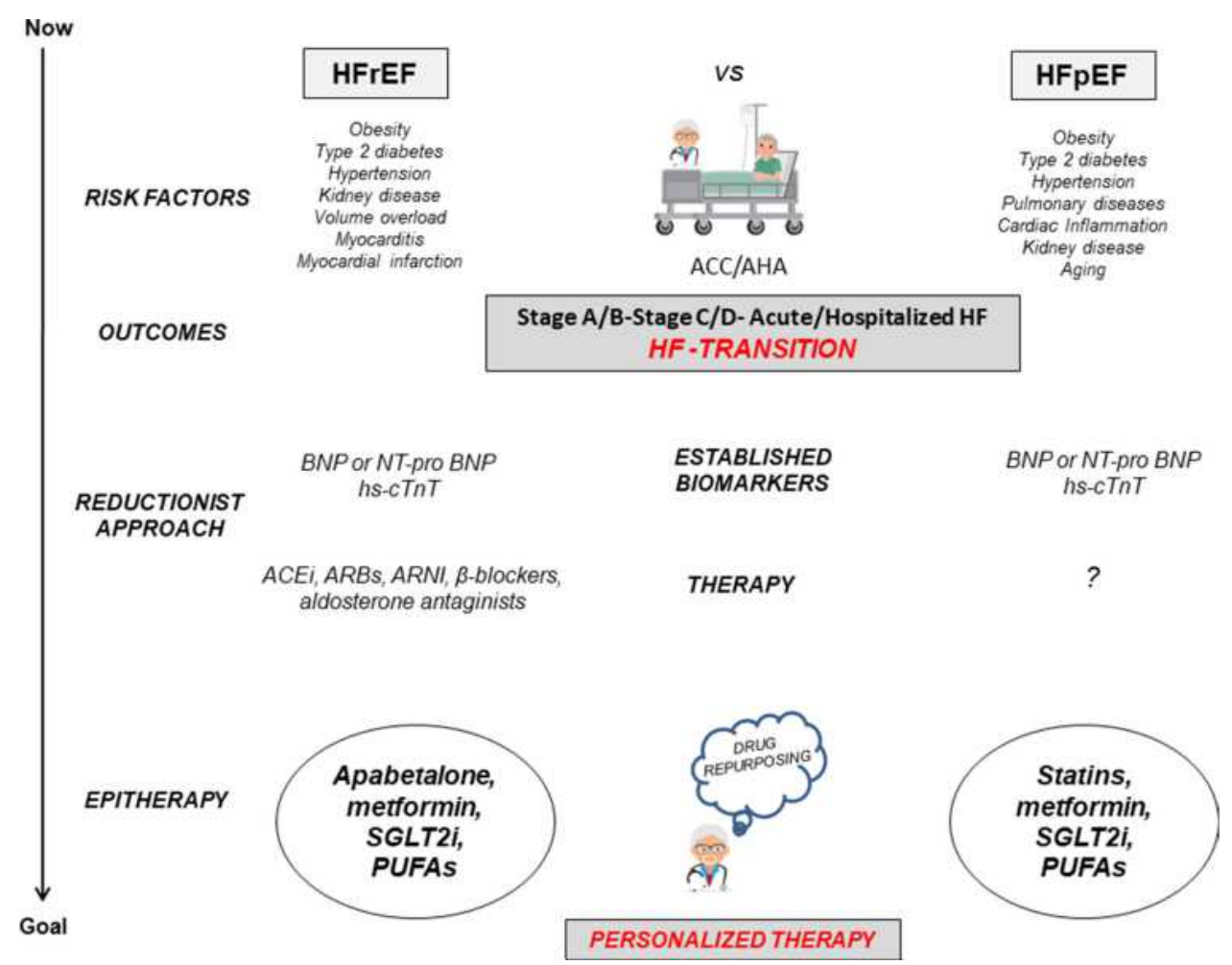

Figure I The possible role of epitherapy in the current framework of HFrEF and HFpEF management. The unstable transition state from the ACC/AHA Stage A/B to Stage C/D-Acute/Hospitalized HF is the key point in the treatment of HFrEF and HFpEF. The epitherapy, mainly apabetalone, statins, metformin, SGLT2i, and PUFAs in addition to the standard of the care may improve personalized therapy of affected patients.

Abbreviations: HFpEF, heart failure with preserved ejection fraction; HFrEF, heart failure with reduced ejection fraction; SGLT2i, sodium glucose co-transporter 2 inhibitors; PUFAs, polyunsaturated fatty acids.

development of major CVDs which may lead to differential HF clinical phenotypes. ${ }^{6-14}$ Of note, the reversible nature of epigenetic-sensitive changes has been translated in the clinical management of specific hematological malignancies with the approval by the Food and Drug Administration (FDA) of some "epidrugs", such as decitabine (Dacogen) and azacitidine (Vidaza), as DNA methylation inhibitors, as well as vorinostat (Zolinza), belinostat (Beleodaq), romidepsin (Istodax), and panobinostat (Farydak), as histone deacetylase inhibitors (HDACi) ${ }^{15}$ Epidrugs are now providing a novel vision for personalized therapy of HF and heart transplantation, opening up novel options for management of the affected patients. ${ }^{15-18}$ At molecular level, we can classify the epidrugs in: "direct epidrugs" [eg, the bromodomain and extra-terminal (BET) protein inhibitor apabetalone]; and repurposed drugs with potential, indirect (non-classical) epigenetic-oriented interference by which they may exert cardioprotective functions [eg, hydralazine, metformin, statins, and sodium-glucose cotransporter-2 inhibitors (SGLT2i)] or nutraceutical compounds [eg, omega-3 polyunsaturated fatty acids (PUFAs)]. Encouraging results are coming from large randomized trials evaluating the putative beneficial effects of combining epidrugs with the conventional therapy in patients with HF. ${ }^{14-22}$ Our goal is to update on the emerging epigeneticbased strategies which may be useful in the prevention and treatment of HFrEF and HFpEF (Figure 1).

\section{Apabetalone: The First and Unique "Direct Epidrug" for Management of HF}

\section{Apabetalone Can Improve Cardiac Function: A Focus on Molecular Mechanisms}

The bromodomain and extra terminal domain (BET) proteins, including the ubiquitous BRD2, BRD3, BRD4, and the testisrestricted BRDT, are epigenetic readers (via bromodomains) existing in the form of nuclear multidomain docking platforms which control the cell-specific activation of gene expression profiles. ${ }^{23}$ Experimental data demonstrated that BETs regulate vascular cells, cardiac myocytes, and inflammatory cells, ${ }^{24}$ and their activity may be extended to the regulation of calcification, thrombosis, as well as lipid and lipoprotein metabolism, all of which participate in atherogenesis. ${ }^{25-27}$ In particular, BRD4 facilitated the expression of multiple proinflammatory and 
proatherosclerotic targets involved in thrombosis, leukocyte adhesion, and endothelial barrier function, thus identifying BRD4 as a possible therapeutic target in CVD setting. ${ }^{24}$ The quinazolone (RVX-208), known as apabetalone, is a derivative of the plant polyphenol resveratrol. Apabetalone acts as a direct epidrug by selectively targeting the BET family member BRD4 to block its interaction with acetylated lysines located in histones. ${ }^{28}$ Apabetalone-BRD4 binding can impact cholesterol levels and inflammation; in fact, apabetalone stimulates ApoA-I gene expression and increases high-density lipoprotein (HDL). ${ }^{29,30}$ Besides, apabetalone may attenuate the development of cardiac hypertrophy ${ }^{31}$ and cardiac fibrosis, ${ }^{32}$ suggesting novel options for the management of HF.

\section{First Results from BETonMACE Clinical Trial}

Resverlogix developed apabetalone (RVX-208), a first-inclass, orally available, small molecule for the treatment of atherosclerosis and associated CVDs. ${ }^{20}$ BETonMACE (NCT02586155) is the first Phase 3 clinical trial evaluating the cardiovascular efficacy and safety of apabetalone. ${ }^{22}$ Recent results from the BETonMACE study have demonstrated that apabetalone is associated with a reduction in first HF hospitalization and cardiovascular death in patients with type 2 diabetes and recent acute coronary syndrome as compared to controls (placebo-treated patients). ${ }^{22}$ Additionally, a significant increase in HDL and a decrease in alkaline phosphatase levels have been observed following 24 weeks of apabetalone treatment as compared to the placebo group. ${ }^{22}$ However, investigators were unable to make a distinction between HF in the setting of preserved or reduced ejection fraction. Thus, further clinical trials should be designed to evaluate the putative beneficial effects of apabetalone in HFrEF and HFpEF, separately.

\section{Repurposed Drugs with a Potential "Indirect" Epigenetic-Oriented Interference for Management of HF Indirect Epidrugs Can Improve Vascular Health and Cardiac Remodeling: Insights from Preclinical Models}

Preclinical studies demonstrated that pharmacological HDACi, ${ }^{33-36}$ BET inhibitors, ${ }^{31,37}$ and DNA methylation inhibitors $^{38}$ can attenuate cardiac remodeling (cardiomyocyte hypertrophy and fibrosis). Although not originally developed as epidrugs, hydralazine (anti-hypertensive drug), metformin, and SGLT2i (anti-diabetic drugs), statins (anti-dyslipidemic drugs), and PUFAs (nutraceuticals) might have downstream epigenetic-oriented effects in cardiac cells. Hydralazine, for example, lowers blood pressure by a direct relaxation of vascular smooth muscle; additionally, it may reduce DNA methylation and improve cardiac function through increasing sarcoplasmic reticulum Ca2+-ATPase (SERCA2a) and modulating calcium homeostasis in cardiomyocytes. ${ }^{39}$ Statins are used as a first-line treatment to decrease serum cholesterol levels in dyslipidemic patients and as primary and secondary prophylaxis against atherosclerosis and associated CVDs. ${ }^{6}$ Many of their non-classical pleiotropic properties relevant for endothelial health are mediated by epigenetic mechanisms which improve blood flow, decrease LDL oxidation, enhance atherosclerotic plaque stability and decrease proliferation of vascular smooth muscle cells and platelet aggregation. ${ }^{6}$ Metformin is a first-line drug in the treatment of overweight and obese type 2 diabetic patients. ${ }^{10}$ Mechanistically, metformin may also have epigenetic-oriented effects through activating the AMP-activated protein kinase (AMPK) which, in turn, can phosphorylate and inhibit epigenetic enzymes such as histone acetyltransferases (HATs), class II HDAC, and DNA methyltransferases (DNMTs). ${ }^{40}$ Both metformin $^{41,42}$ and statins $^{43,44}$ may reduce cardiac fibrosis; however, whether their beneficial effects are mediated by epigenetic-oriented responses has yet to be demonstrated. Furthermore, SGLT2i are a new group of oral drugs used for treating type 2 diabetes and its cardiovascular/renal complications. ${ }^{45}$ Animal models have demonstrated that empagliflozin ${ }^{46,47}$ and dapagliflozin ${ }^{48}$ may improve hemodynamics in HF by increasing renal protection and cardiac fibrosis. Interestingly, inflammation and glucotoxicity (AGE/RAGE signaling) were epigenetically prevented by empagliflozin; ${ }^{49}$ this observation has provided insights about mechanisms by which SGLT2i can reduce cardiovascular mortality in man (EMPA-REG trial). ${ }^{50}$

\section{Indirect Epidrugs in Clinical Trials for Management of Patients with HFpEF}

An effective therapy for HFpEF has yet to be established. Hydralazine is frequently used in HFrEF, and represents a potential DNA methylation inhibitor. ${ }^{39}$ DNA methylation is the most studied direct epigenetic change with potential clinical implications in major CVDs and the development of $\mathrm{HF}^{7,14}$ This epigenetic signature mainly involves methylation of $\mathrm{CpG}$ islands in the gene promoters leading to a specific longterm silencing of gene expression. ${ }^{7,14}$ A completed Phase 2 clinical trial (NCT01516346) evaluated the effect of prolonged therapy (24 weeks) with isosorbide dinitrate (ISDN) \pm 
hydralazine on arterial wave reflections (primary endpoint) as well as left ventricular (LV) mass, fibrosis and diastolic function, and exercise capacity (6-minute walk test) in patients with HFpEF, New York Heart Association (NYHA) Class II-IV symptoms, and standard therapy as defined by ACEi, ARB, beta-blockers, or calcium channel blockers (CCBs).${ }^{51}$ Results from this trial reported that ISDN, with or without hydralazine, had deleterious effects on reflection magnitude, LV remodeling, or submaximal exercise thus not supporting their routine use in patients with $\mathrm{HFpEF}{ }^{51}$

Metformin has been associated with a reduced mortality in patients with HFpEF, even if female gender was associated with worse outcomes. ${ }^{52}$ Recently, it has been observed that a long-term treatment with metformin can improve LV diastolic function and hypertrophy, decrease the incidence of new-onset HFpEF, and delay disease progression in patients with type 2 diabetes and hypertension. ${ }^{53}$ Besides, a prospective phase 2 clinical trial (NCT03629340) is testing the therapeutic efficacy of metformin in patients with pulmonary hypertension and HFpEF by evaluating exercise hemodynamics, functional capacity, skeletal muscle signaling, and insulin sensitivity. However, results have not been published. A recent study based on the JASPER registry, a multicenter, observational, prospective cohort of Japanese patients aged $\geq 20$ years requiring hospitalization for acute $\mathrm{HFpEF}$ has reported that the use of statins could reduce mortality in affected patients without coronary heart disease. ${ }^{54}$ Furthermore, the use of statins was associated with improved clinical outcomes in patients with HFpEF but not in patients with HFrEF (or mid-range ejection fraction). ${ }^{55} \mathrm{~A}$ reduced rate of major adverse cardiac events, cardiovascular death and all-cause mortality was associated with SGLT2i treatment in both HFpEF and HFrEF patients as compared to placebo. ${ }^{56,57}$ However, the observed cardiovascular and renal benefits cannot be fully explained by improvement in risk factors (such as glycemia, blood pressure or dyslipidemias) suggesting that other molecular mechanisms may explain the cardiovascular benefits. ${ }^{56}$ Interestingly, the SGLT2i-related epigenetic interference may arise from their ability to increase the circulating and tissue levels of $\beta$-hydroxybutyrate, a specific molecule able to generate a pattern of histone modifications (known as $\beta$-hydroxybutyrylation) which are associated with the beneficial effects of fasting. ${ }^{58}$ Besides, the DELIVER (NCT03619213) multicenter, randomized, double-blind, placebo-controlled study of $6263 \mathrm{HFpEF}$ patients will evaluate the effect of dapagliflozin $10 \mathrm{mg}$
(1 per day) as compared to placebo in addition to the standard of care in order to reduce the composite of cardiovascular death or HF events. However, results have not yet been published.

\section{Indirect Epidrugs in Clinical Trials for Management of Patients with HFrEF}

The use of metformin has been generally considered a contraindication in HFrEF patients owing the potential risk of lactic acidosis; however, recent evidence has reported that metformin can provide beneficial effects in reducing the risk of incident $\mathrm{HF}$ and mortality in diabetic patients. ${ }^{59-61}$ A completed, observational clinical trial (NCT03546062) has recently performed the evaluation of seriated cardiac biopsies from healthy implanted hearts in type 2 diabetes recipients during 12-month follow-up upon heart transplantation. ${ }^{21}$ Even if the intra-cardiomyocyte lipid accumulation in type 2 diabetes recipients may start in the early stages after heart transplantation, metformin therapy could reduce lipid accumulation independently of immunosuppressive therapy. ${ }^{21}$ The DANHEART trial (NCT03514108), a multicenter, randomized, double-blind, placebo-controlled study in 1500 patients with HFrEF will evaluate: 1) whether hydralazine-isosorbide dinitrate as compared to placebo may reduce the incidence of death and HF hospitalization, and 2) if metformin as compared to placebo may reduce the incidence of death, worsening of $\mathrm{HF}$, acute myocardial infarction, and stroke in patients with diabetes or prediabetes. Two large randomized trials demonstrated that statins did not have beneficial effects in management of patients with HFrEF. ${ }^{62,63}$ Specifically, the CORONA phase 3 trial randomized more than 5000 patients with ischemic HFrEF to rosuvastatin as compared to placebo resulting in no benefits on the primary endpoints, as death from cardiovascular causes, nonfatal myocardial infarction, and nonfatal stroke. ${ }^{62}$ According to CORONA trial, the GISSIHF study randomized almost 5000 patients with clinically apparent HF of any cause to rosuvastatin as compared to placebo and observed no benefits on the primary endpoints, as all-cause death or cardiovascular hospitalization. ${ }^{63}$ However, it is needed to highlight that both trials demonstrated that statins are safe in HF patients. In contrast with the previous evidence, the trial based on the Swedish Heart Failure Registry (21,864 patients with HFrEF, of whom 10,345 were treated with statins) reported an association between the use of statins and improved outcomes, as all-cause mortality, cardiovascular mortality, HF hospitalization, and combined all-cause mortality or cardiovascular hospitalization, especially in patients with 


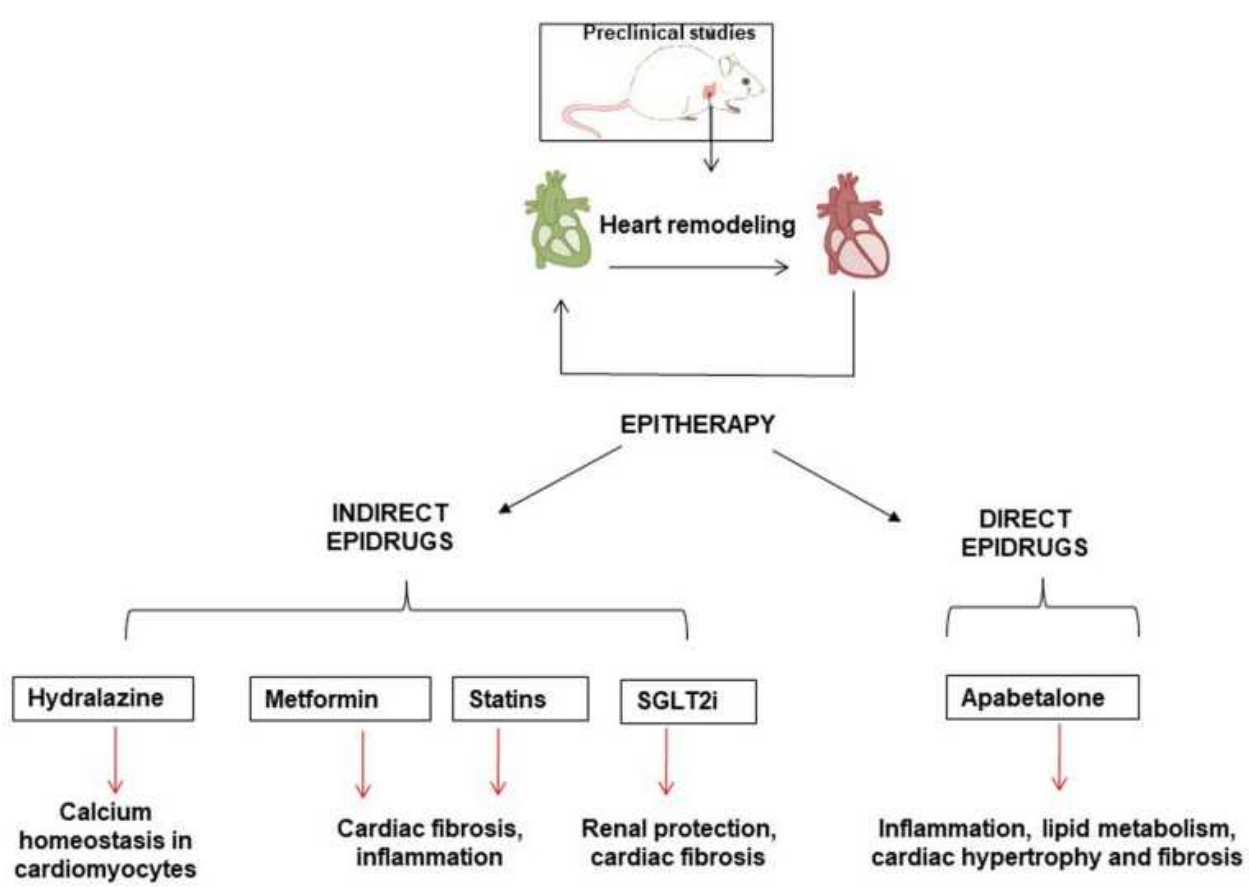

Figure 2 Direct and indirect epigenetic drugs in preclinical models of HF. Cardiac remodeling includes different pathological phenotypes and each type of drug can selectively improve inflammation, cardiac fibrosis and hypertrophy, calcium homeostasis, and lipid metabolism.

Abbreviations: HF, heart failure; SGLT2i, sodium glucose co-transporter 2 inhibitors.

ischemic HF. ${ }^{64}$ Thus, further randomized controlled trials focused on ischemic HF may be warranted. Omega-3 polyunsaturated fatty acids (PUFAs), mainly eicosapentaenoic acid (EPA) and docosahexaenoic acid (DHA), are key players in modulating inflammatory process by limiting leucocyte chemotaxis, adhesion molecule expression, leucocyteendothelium interaction as well as $\mathrm{T}$ cell reactivity. ${ }^{65} \mathrm{EPA}$ and DHA are mainly gained from marine food consumption and large population-based studies have shown that Mediterranean diet with PUFA supplementation may aid to prevent CVDs owing to their ability in promoting the release of nitric oxide from endothelial cells and decreasing serum levels of triglycerides ${ }^{66}$ Recent evidence has indicated that PUFAs can significantly affect the cellular epigenome mainly thought DNA methylation-sensitive mechanisms. ${ }^{67,68}$ The GISSI multicenter, double-blind trial enrolled 6975 HF patients (New York Heart Association class II-IV, irrespective of cause and LV ejection fraction) and randomized them to low dose $(0.84 \mathrm{~g}$ per day) of PUFAs as compared to placebo. PUFAs supplementation reduced risk for total mortality and HF hospitalization when added to standard therapy. ${ }^{19}$ Furthermore, in the OMEGA-REMODEL trial, high-dose of PUFAs ( $3.4 \mathrm{~g}$ per day) for 6 months post-myocardial infarction reduced infarct size and non-infarct myocardial fibrosis as well as improved ventricular systolic function. ${ }^{69}$ Taken together, these results suggest that PUFAs may aid to prevent HFrEF. More recently, the MESA longitudinal trial including 6562 participants 45 to 84 years has demonstrated that higher plasma levels of EPA were significantly associated with reduced risk both in HFpEF and HFrEF. ${ }^{70}$

\section{Concluding Remarks}

Although the possibility of improving the HF standard of care with epidrugs is still in its infancy, the BETonMACE study has provided promising results about the use of apabetalone in reducing hospitalization and cardiovascular death. Preclinical models of cardiac remodeling demonstrated that metformin, statins, SGLT2i, and PUFAs ${ }^{41-48}$ can improve vascular health and cardiac fibrosis by modulating specific molecular pathways, and, in part, through downstream epigenetic interference, especially for hydralazine ${ }^{39}$ and empagliflozin (Figure 2). ${ }^{49}$ Of note, metformin and SGLT2i can impact on the "epigenetic memory" phenomenon. This latter suggests that an early glycemia normalization can arrest hyperglycemiainduced epigenetic processes associated with enhanced oxidative stress and glycation of cellular proteins and lipids. ${ }^{71,72}$ In parallel, an increasing number of clinical trials is evaluating the putative beneficial repurposing of metformin, statins, SGLT2i, and PUFAs in patients with HFpEF and/or HFrEF; ${ }^{19,62-64,69,73-75}$ however, despite experimental 
evidence, none of these trials evaluated their potential epigenetic effects involved in improving the cardiac function. This gap should be overcome to improve personalized therapy of patients with HF. Thus, further randomized trials are needed to clarify whether apabetalone, as well as non-canonical repurposed epidrugs, will really be able to save failing hearts in different HF clinical phenotypes or prevent irreversible damages in high-risk patients. In this context, Network Medicine approaches may help to evaluate a possible repurposing of epidrugs in patients with major CVDs. ${ }^{15,76,77}$

\section{Funding}

This work was supported by PRIN2017F8ZB89 from "Italian Ministry of University and Research (MIUR)" (PI Prof Napoli) and Ricerca Corrente (RC) 2019 from "Italian Ministry of Health" (PI Prof. Napoli).

\section{Disclosure}

The authors report no conflicts of interest in this work.

\section{References}

1. Gronda E, Sacchi S, Benincasa G, et al. Unresolved issues in left ventricular postischemic remodeling and progression to heart failure. J Cardiovasc Med (Hagerstown). 2019;20:640-649. doi:10.2459/ JCM.0000000000000834.

2. Gronda E, Vanoli E, Sacchi S, et al. Risk of heart failure progression in patients with reduced ejection fraction: mechanisms and therapeutic options. Heart Fail Rev. 2020;25(2):295-303. doi:10.1007/s10741019-09823-z

3. Sokos GG, Raina A. Understanding the early mortality benefit observed in the PARADIGM-HF trial: considerations for the management of heart failure with sacubitril/valsartan. Vasc Health Risk Manag. 2020;16:41-51. doi:10.2147/VHRM.S197291

4. Cacciatore F, Amarelli C, Maiello C, et al. Sacubitril/valsartan in patients listed for heart transplantation: effect on physical frailty. ESC Heart Fail. 2020;7:757-762. doi:10.1002/ehf2.12610.

5. Clark KAA, Velazquez EJ. Heart failure with preserved ejection fraction: time for a reset. JAMA. 2020;324:1506-1508. doi:10.1001/ jama.2020.15566.

6. Schiano C, Benincasa G, Franzese M, et al. Epigenetic-sensitive pathways in personalized therapy of major cardiovascular diseases. Pharmacol Ther. 2020;210:107514. doi:10.1016/j. pharmthera.2020.107514.

7. Schiano $C$, Benincasa $G$, Infante $T$, et al. Integrated analysis of DNA methylation profile of HLA-G gene and imaging in coronary heart disease: pilot study. PLoS One. 2020;15:e0236951. doi:10.1371/journal.pone. 0236951 .

8. Benincasa G, Cuomo O, Vasco M, et al. Epigenetic-sensitive challenges of cardiohepatic interactions: clinical and therapeutic implications in heart failure patients. Eur J Gastroenterol Hepatol. 2020. doi:10.1097/MEG.0000000000001867.

9. Benincasa G, Franzese M, Schiano C, et al. DNA methylation profiling of CD04+/CD08+ T cells reveals pathogenic mechanisms in increasing hyperglycemia: PIRAMIDE pilot study. Ann Med Surg (Lond). 2020;60:218-226. doi:10.1016/j.amsu.2020.10.016.
10. Napoli C, Benincasa G, Schiano C, et al. Differential epigenetic factors in the prediction of cardiovascular risk in diabetic patients. Eur Heart J Cardiovasc Pharmacother. 2020;6:239-247. doi:10.1093/ehjcvp/pvz062.

11. Napoli C, Coscioni E, de Nigris F, et al. Emergent expansion of clinical epigenetics in patients with cardiovascular diseases. Curr Opin Cardiol. 2021;36(3):295-300. doi:10.1097/ HCO.0000000000000843.

12. Infante T, Forte E, Schiano C, et al. Evidence of association of circulating epigenetic-sensitive biomarkers with suspected coronary heart disease evaluated by Cardiac Computed Tomography. PLoS One. 2019;14:e210909. doi:10.1371/journal.pone.0210909.

13. de Nigris F, Cacciatore F, Mancini FP, et al. Epigenetic hallmarks of fetal early atherosclerotic lesions in humans. JAMA Cardiol. 2018;3:1184-1191. doi:10.1001/jamacardio.2018.3546.

14. Napoli C, Benincasa G, Donatelli F, et al. Precision medicine in distinct heart failure phenotypes: focus on clinical epigenetics. $\mathrm{Am}$ Heart J. 2020;224:113-128. doi:10.1016/j.ahj.2020.03.007.

15. Sarno F, Benincasa G, List M, et al. Clinical epigenetics settings for cancer and cardiovascular diseases: real-life applications of network medicine at the bedside. Clin Epigenetics. 2021;13:66. doi:10.1186/ s13148-021-01047-z.

16. Grimaldi V, Vietri MT, Schiano C, et al. Epigenetic reprogramming in atherosclerosis. Curr Atheroscler Rep. 2014;17:476. doi:10.1007/ s11883-014-0476-3.

17. Sabia C, Picascia A, Grimaldi V, et al. The epigenetic promise to improve prognosis of heart failure and heart transplantation. Transplant Rev (Orlando). 2017;31:249-256. doi:10.1016/j.trre.2017.08.004.

18. Vasco M, Benincasa G, Fiorito C, et al. Clinical epigenetics and acute/chronic rejection in solid organ transplantation: an update. Transplant Rev (Orlando). 2021;35:100609. doi:10.1016/j. trre.2021.100609.

19. Tavazzi L, Maggioni AP, Marchioli R, et al. Effect of n-3 polyunsaturated fatty acids in patients with chronic heart failure (the GISSI-HF trial): a randomised, double-blind, placebo-controlled trial. Lancet. 2008;372:1223-1230. doi:10.1016/S0140-6736(08)61239-8.

20. Ray KK, Nicholls SJ, Buhr KA, et al. Effect of apabetalone added to standard therapy on major adverse cardiovascular events in patients with recent acute coronary syndrome and type 2 diabetes: a randomized clinical trial. JAMA. 2020;323:1565-1573. doi:10.1001/jama.2020.3308.

21. Marfella R, Amarelli C, Cacciatore F, et al. Lipid accumulation in hearts transplanted from nondiabetic donors to diabetic recipients. $\mathrm{J} \mathrm{Am} \mathrm{Coll}$ Cardiol. 2020;75:1249-1262. doi:10.1016/j.jacc.2020.01.018.

22. Nicholls SJ, Schwartz GG, Buhr KA, et al. Apabetalone and hospitalization for heart failure in patients following an acute coronary syndrome: a prespecified analysis of the BETonMACE study. Cardiovasc Diabetol. 2021;20:13. doi:10.1186/s12933-020-01199-x.

23. Gaucher J, Boussouar F, Montellier E, et al. Bromodomain-dependent stage-specific male genome programming by Brdt. EMBO J. 2012;31:3809-3820. doi:10.1038/emboj.2012.233.

24. Borck PC, Guo LW, Plutzky J. BET epigenetic reader proteins in cardiovascular transcriptional programs. Circ Res. 2020;126:1190-1208. doi:10.1161/CIRCRESAHA.120.315929.

25. Tonini C, Colardo M, Colella B, et al. Inhibition of bromodomain and extraterminal domain (BET) proteins by JQ1 unravels a novel epigenetic modulation to control lipid homeostasis. Int $J \mathrm{Mol}$ Sci. 2020;21:1297. doi:10.3390/ijms21041297.

26. Nicodeme E, Jeffrey KL, Schaefer U, et al. Suppression of inflammation by a synthetic histone mimic. Nature. 2010;468:1119-1123. doi:10.1038/nature09589.

27. Dey A, Yang W, Gegonne A, et al. BRD4 directs hematopoietic stem cell development and modulates macrophage inflammatory responses. EMBO J. 2019;38:e100293. doi:10.15252/ embj.2018100293. 
28. Picaud S, Wells C, Felletar I, et al. RVX-208, an inhibitor of BET transcriptional regulators with selectivity for the second bromodomain. Proc Natl Acad Sci U S A. 2013;110:19754-19759. doi:10.1073/pnas.1310658110.

29. McLure KG, Gesner EM, Tsujikawa L, et al. RVX-208, an inducer of ApoA-I in humans, is a BET bromodomain antagonist. PLoS One. 2013;8:e83190. doi:10.1371/journal.pone.0083190.

30. Bailey D, Jahagirdar R, Gordon A, et al. RVX-208: a small molecule that increases apolipoprotein A-I and high-density lipoprotein cholesterol in vitro and in vivo. $J$ Am Coll Cardiol. 2010;55:2580-2589. doi:10.1016/j.jacc.2010.02.035.

31. Anand P, Brown JD, Lin CY, et al. BET bromodomains mediate transcriptional pause release in heart failure. Cell. 2013;154:569-582. doi:10.1016/j.cell.2013.07.013.

32. Song S, Liu L, Yu Y, et al. Inhibition of BRD4 attenuates transverse aortic constriction- and TGF- $\beta$-induced endothelial-mesenchymal transition and cardiac fibrosis. J Mol Cell Cardiol. 2019;127:83-96. doi:10.1016/j.yjmcc.2018.12.002.

33. Ooi JY, Tuano NK, Rafehi H, et al. HDAC inhibition attenuates cardiac hypertrophy by acetylation and deacetylation of target genes. Epigenetics. 2015;10:418-430. doi:10.1080/1559229 4.2015.1024406.

34. Ferguson BS, McKinsey TA. Non-sirtuin histone deacetylases in the control of cardiac aging. $J$ Mol Cell Cardiol. 2015;83:14-20. doi:10.1016/j.yjmcc.2015.03.010.

35. Chen Y, Du J, Zhao YT, et al. Histone deacetylase (HDAC) inhibition improves myocardial function and prevents cardiac remodeling in diabetic mice. Cardiovasc Diabetol. 2015;14:99. doi:10.1186/ s12933-015-0262-8.

36. Zhang CL, McKinsey TA, Chang S, et al. Class II histone deacetylases act as signal-responsive repressors of cardiac hypertrophy. Cell. 2002;110(4):479-488. doi:10.1016/s0092-8674(02)00861-9

37. Spiltoir JI, Stratton MS, Cavasin MA, et al. BET acetyl-lysine binding proteins control pathological cardiac hypertrophy. $J$ Mol Cell Cardiol. 2013;63:175-179. doi:10.1016/j.yjmcc.2013.07.017.

38. Russell-Hallinan A, Neary R, Watson CJ, et al. Repurposing from oncology to cardiology: low-dose 5-azacytidine attenuates pathological cardiac remodeling in response to pressure overload injury. J Cardiovasc Pharmacol Ther. 2020:107424842097923. doi:10.1177/1074248420979235

39. Kao YH, Cheng CC, Chen YC, et al. Hydralazine-induced promoter demethylation enhances sarcoplasmic reticulum $\mathrm{Ca} 2+-$ ATPase and calcium homeostasis in cardiac myocytes. Lab Invest. 2011;91:1291-1297. doi:10.1038/labinvest.2011.92.

40. Bridgeman SC, Ellison GC, Melton PE, et al. Epigenetic effects of metformin: from molecular mechanisms to clinical implications. Diabetes Obes Metab. 2018;20(7):1553-1562. doi:10.1111/ dom. 13262.

41. Xiao H, Ma X, Feng W, et al. Metformin attenuates cardiac fibrosis by inhibiting the TGFbeta1-Smad3 signalling pathway. Cardiovasc Res. 2010;87:504-513. doi:10.1093/cvr/cvq066.

42. Zhao Q, Song W, Huang J, et al. Metformin decreased myocardial fibrosis and apoptosis in hyperhomocysteinemia -induced cardiac hypertrophy. Curr Res Transl Med. 2021;69:103270. doi:10.1016/j. retram.2020.103270.

43. Oesterle A, Laufs U, Liao JK. Pleiotropic effects of statins on the cardiovascular system. Circ Res. 2017;120:229-243. doi:10.1161/ CIRCRESAHA.116.308537.

44. Sun F, Duan W, Zhang Y, et al. Simvastatin alleviates cardiac fibrosis induced by infarction via up-regulation of TGF- $\beta$ receptor III expression. Br J Pharmacol. 2015;172:3779-3792. doi:10.1111/bph.13166.

45. Gronda E, Jessup M, Iacoviello M, et al. Glucose metabolism in the kidney: neurohormonal activation and heart failure development. $J$ Am Heart Assoc. 2020;9(23):e018889. doi:10.1161/JAHA.12 0.018889 .
46. Lee HC, Shiou YL, Jhuo SJ, et al. The sodium-glucose co-transporter 2 inhibitor empagliflozin attenuates cardiac fibrosis and improves ventricular hemodynamics in hypertensive heart failure rats. Cardiovasc Diabetol. 2019;18:45. doi:10.1186/s12933019-0849-6.

47. Li C, Zhang J, Xue M, et al. SGLT2 inhibition with empagliflozin attenuates myocardial oxidative stress and fibrosis in diabetic mice heart. Cardiovasc Diabetol. 2019;18:15. doi:10.1186/s12933-019-0816-2.

48. Arow M, Waldman M, Yadin D, et al. Sodium-glucose cotransporter 2 inhibitor Dapagliflozin attenuates diabetic cardiomyopathy. Cardiovasc Diabetol. 2020;19:7. doi:10.1186/ s12933-019-0980-4.

49. Steven S, Oelze M, Hanf A, et al. The SGLT2 inhibitor empagliflozin improves the primary diabetic complications in ZDF rats. Redox Biol. 2017;13:370-385. doi:10.1016/j.redox.2017.06.009.

50. Zinman B, Lachin JM, Inzucchi SE. Empagliflozin, cardiovascular outcomes, and mortality in type 2 Diabetes. $N$ Engl $J$ Med. 2016;374:1094. doi:10.1056/NEJMc1600827

51. Zamani P, Akers S, Soto-Calderon H, et al. Isosorbide Dinitrate, with or without hydralazine, does not reduce wave reflections, left ventricular hypertrophy, or myocardial fibrosis in patients with heart failure with preserved ejection fraction. $J$ Am Heart Assoc. 2017;6:e004262. doi:10.1161/JAHA.116.004262.

52. Halabi A, Sen J, Huynh Q, et al. Metformin treatment in heart failure with preserved ejection fraction: a systematic review and meta-regression analysis. Cardiovasc Diabetol. 2020;19(1):124. doi:10.1186/s12933-020-01100-w.

53. Gu J, Yin ZF, Zhang JF, et al. Association between long-term prescription of metformin and the progression of heart failure with preserved ejection fraction in patients with type 2 diabetes mellitus and hypertension. Int J Cardiol. 2020;306:140-145. doi:10.1016/j. ijcard.2019.11.087.

54. Marume K, Takashio S, Nagai T, et al. Effect of statins on mortality in heart failure with preserved ejection fraction without coronary artery disease. Report from the JASPER Study. Circ J. 2019;83:357-367. doi:10.1253/circj.CJ-18-0639.

55. Lee MS, Duan L, Clare R, et al. Comparison of effects of statin use on mortality in patients with heart failure and preserved versus reduced left ventricular ejection fraction. $\mathrm{Am} J$ Cardiol. 2018;122:405-412. doi:10.1016/j.amjcard.2018.04.027.

56. Lam CSP, Chandramouli C, Ahooja V, Verma S. SGLT-2 inhibitors in heart failure: current management, unmet needs, and therapeutic prospects. J Am Heart Assoc. 2019;8:e13389. doi:10.1161/ JAHA.119.013389.

57. Zannad F, Ferreira JP, Pocock SJ, et al. SGLT2 inhibitors in patients with heart failure with reduced ejection fraction: a meta-analysis of the EMPEROR-Reduced and DAPA-HF trials. Lancet. 2020;396:819-829. doi:10.1016/S0140-6736(20)31824-9.

58. Nishitani S, Fukuhara A, Shin J, et al. Metabolomic and microarray analyses of adipose tissue of dapagliflozin-treated mice, and effects of 3-hydroxybutyrate on induction of adiponectin in adipocytes. Sci Rep. 2018;8:8805. doi:10.1038/s41598-018-27181-y.

59. Wong AK, AlZadjali MA, Choy AM, et al. Insulin resistance: a potential new target for therapy in patients with heart failure. Cardiovasc Ther. 2008;26:203-213. doi:10.1111/j.17555922.2008.00053.x.

60. Pantalone KM, Kattan MW, Yu C, et al. The risk of developing coronary artery disease or congestive heart failure, and overall mortality, in type 2 diabetic patients receiving rosiglitazone, pioglitazone, metformin, or sulfonylureas: a retrospective analysis. Acta Diabetol. 2009;46:145-154. doi:10.1007/s00592-008-0090-3.

61. Papanas N, Maltezos E, Mikhailidis DP. Metformin and heart failure: never say never again. Expert Opin Pharmacother. 2012;13:1-8. doi:10.1517/14656566.2012.638283. 
62. Kjekshus J, Apetrei E, Barrios V, et al. Rosuvastatin in older patients with systolic heart failure. $N$ Engl J Med. 2007;357:2248-2261. doi:10.1056/NEJMoa0706201.

63. Tavazzi L, Maggioni AP, Marchioli R, et al. Effect of rosuvastatin in patients with chronic heart failure (the GISSI-HF trial): a randomised, double-blind, placebo-controlled trial. Lancet. 2008;372:1231-1239. doi:10.1016/S0140-6736(08)61240-4.

64. Alehagen U, Benson L, Edner M, et al. Association between use of statins and outcomes in heart failure with reduced ejection fraction: prospective propensity score matched cohort study of 21864 patients in the Swedish Heart Failure Registry. Circ Heart Fail. 2015;8:252-260. doi:10.1161/CIRCHEARTFAILURE.114.001730.

65. Calder PC. Omega-3 polyunsaturated fatty acids and inflammatory processes: nutrition or pharmacology? $\mathrm{Br} J$ Clin Pharmacol. 2013;75:645-662. doi:10.1111/j.1365-2125.2012.04374.x.

66. Mohebi-Nejad A, Bikdeli B. Omega-3 supplements and cardiovascular diseases. Tanaffos. 2014;13:6-14.

67. Burdge GC, Lillycrop KA. Fatty acids and epigenetics. Curr Opin Clin Nutr Metab Care. 2014;17:156-161. doi:10.1097/ MCO.0000000000000023.

68. de la Rocha C, Pérez-Mojica JE, León SZ, et al. Associations between whole peripheral blood fatty acids and DNA methylation in humans. Sci Rep. 2016;6:25867. doi:10.1038/srep25867.

69. Heydari B, Abdullah S, Pottala JV, et al. Effect of omega-3 acid ethyl esters on left ventricular remodeling after acute myocardial infarction: the OMEGA-REMODEL randomized clinical trial. Circulation. 2016;134:378-391. doi:10.1161/CIRCULATIO NAHA.115.019949.
70. Block RC, Liu L, Herrington DM, et al. Predicting risk for incident heart failure with omega-3 fatty acids: from MESA. JACC Heart Fail. 2019;7:651-661. doi:10.1016/j.jchf.2019.03.008.

71. Berezin A. Metabolic memory phenomenon in diabetes mellitus: achieving and perspectives. Diabetes Metab Syndr. 2016;10:S17683. doi:10.1016/j.dsx.2016.03.016.

72. Sommese L, Benincasa G, Lanza M, et al. Novel epigenetic-sensitive clinical challenges both in type 1 and type 2 diabetes. J Diabetes Complications. 2018;32:1076-1084. doi:10.1016/j. jdiacomp.2018.08.012.

73. Trum M, Wagner S, Maier LS, et al. CaMKII and GLUT1 in heart failure and the role of gliflozins. Biochim Biophys Acta Mol Basis Dis. 2020;1866:165729. doi:10.1016/j.bbadis.2020.165729.

74. Nikolic M, Zivkovic V, Jovic JJ, et al. SGLT2 inhibitors: a focus on cardiac benefits and potential mechanisms. Heart Fail Rev. 2021. doi:10.1007/s10741-021-10079-9.

75. Mohammadzadeh N, Montecucco F, Carbone F, et al. Statins: epidrugs with effects on endothelial health? Eur J Clin Invest. 2020;50: e13388. doi:10.1111/eci.13388.

76. Benincasa G, DeMeo DL, Glass K, Silverman EK, Napoli C. Epigenetics and pulmonary diseases in the horizon of precision medicine: a review. Eur Respir J. 2020 Nov 19:2003406. doi:10.1183/13993003.03406-2020.77.

77. Benincasa G, Marfella R, Della Mura N, et al. Strengths and opportunities of network medicine in cardiovascular diseases. Circ J. 2020 Jan 24;84(2):144-152. doi:10.1253/circj.CJ-19-0879.
Vascular Health and Risk Management

\section{Publish your work in this journal}

Vascular Health and Risk Management is an international, peerreviewed journal of therapeutics and risk management, focusing on concise rapid reporting of clinical studies on the processes involved in the maintenance of vascular health; the monitoring, prevention and treatment of vascular disease and its sequelae; and the involvement of metabolic disorders, particularly diabetes. This journal is indexed on PubMed Central and MedLine. The manuscript management system is completely online and includes a very quick and fair peerreview system, which is all easy to use. Visit http://www.dovepress. com/testimonials.php to read real quotes from published authors. 